\title{
Experimental Flow Investigation of a Truncated Ideal Contour Nozzle
}

\author{
Ralf H. Stark \\ German Aerospace Center, Lampoldshausen, D-74239, Germany \\ Bernd H. Wagner \\ University of Stuttgart, Stuttgart, D-70550, Germany
}

\begin{abstract}
Various tests showed a significant side load peak for low nozzle pressure ratios during engine start-up and shut down phase. DLR Lampoldshausen carried out tests to examine the flow field in a truncated ideal contour nozzle for low NPR. For NPR $<10$ a convex and for NPR > 20 a slight concave shaped Mach disk was found. Its curvature is limited to the centre and its height trend correlates with measured side loads. A concave shaped Mach disk being responsible for re-attached flows at low NPR could be excluded. The experiments were accompanied by numerical simulations of the flow field on various pressure ratios with regards on the shock pattern. The predicted Mach disk shape compares well with the experiments.
\end{abstract}

$\begin{array}{ll}R^{*} & =\text { nozzle throat radius } \\ L & =\text { nozzle length } \\ X & =\text { axial coordinate } \\ Y & =\text { radial coordinate } \\ M & =\text { Mach number } \\ H & =\text { height } \\ p & =\text { pressure } \\ t & =\text { time } \\ a & =\text { ambient } \\ w & =\text { nozzle wall } \\ 0 & =\text { total condition }\end{array}$

\section{Nomenclature}

$\begin{array}{ll}\text { tr } & =\text { triple point } \\ \text { Mach } & =\text { Mach disk } \\ \text { sep } & =\text { incipient separation } \\ \text { NPR } & =\text { nozzle pressure ratio } \\ \text { RMS } & =\text { root mean square } \\ \text { CFD } & =\text { computed fluid dynamics } \\ \text { TIC } & =\text { truncated ideal contour } \\ \text { TOP } & =\text { thrust optimized parabola } \\ \text { MOC } & =\text { method of characteristics } \\ \text { TDK } & =\text { Two-Dimesional Kinetics(TDK91/Pro) } \\ \text { DLR } & =\text { German Aerospace Center }\end{array}$

\section{Introduction}

$\mathrm{T}$

The design of today's launchers has changed from a classical tandem to a parallel configuration and the main stage engine therefore has to fulfill a wider range of operation conditions during ascent from sea-level to high altitude. A significant payload gain can be achieved if the main stage engine features a high specific impulse. A possibility to increase the specific impulse is the usage of high area ratio nozzles. As high area ratio nozzles tend to separated flows during sea level operation, leading to uncontrolled side loads due to the asymmetrical nature of the separation, a serial of tests and CFD investigations deal with separated nozzle flows over the last years.

Various tests showed a significant side load peak for low nozzle pressure ratios during engine start-up and shut down phase. DLR Lampoldshausen carried out tests with different kind of nozzles studying the origin of this peak. ${ }^{6}$ A re-attached flow was identified to be the reason of this side load peak (fig. 17). This flow condition was found to be present in thrust optimized parabolic (TOP) nozzles as well as in truncated ideal contour (TIC) nozzles. Up to here re-attached flows were only observed in TOP nozzles during a flow condition known as restricted shock separation (RSS). Competitive CFD investigations ${ }^{6}$ showed a bowed Mach disk creating a flow pattern comparable to the cap shock pattern, featured by TOP nozzles (fig. 18). But here without an internal shock. Such a pattern could explain a partial redirected flow towards the nozzle wall, resulting in side loads.

Computed bowed Mach disks could also be found in Ref. 8 and 9. An experimental Schlieren image is given in Ref. 4 where a shortened TIC nozzle features a bowed Mach disk. These studies and an intensive discussion within Europe's Flow Separation Control Device Group initiate a test campaign investigating the flow pattern in TIC nozzles for low nozzle pressure ratios (NPR) in more detail. 


\section{Experimental Program}

The experiments were conducted in the cold flow subscale test facility P6.2 at German Aerospace Centre DLR in Lampoldshausen, Germany. The test facility provides two test positions, a vertical high altitude chamber with a diameter of $0.8 \mathrm{~m}$ and a height of $1 \mathrm{~m}$ and a horizontal test rig with uninfluenced ambient flow. ${ }^{7}$ As a media dry gaseous nitrogen is used, stored in high pressure tanks.

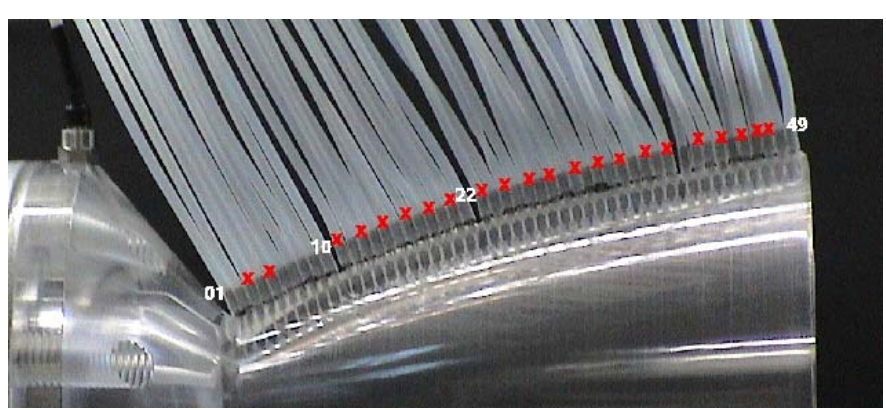

Figure 1. Acrylic glass TIC nozzle with transducer ports.

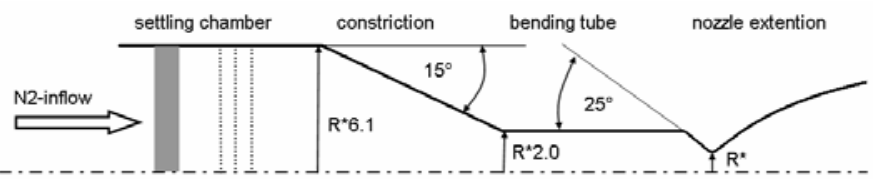

Figure 2. Sketch of horizontal test section.

\section{A. Test Bench Setup and Model}

The model used was a truncated ideal contour nozzle made of acrylic glass with a throat diameter of $0.02 \mathrm{~m}$, a maximum overall length of $0.12 \mathrm{~m}$ and a design Mach number of 5.15, as described in detail in Ref. 3. The model was equipped with 50 pressure transducer ports in stream wise direction with a spacing of $2.5 \mathrm{~mm}$, starting in the nozzle's throat. In the experimental setup the nozzle was mounted on the horizontal test position, $1.2 \mathrm{~m}$ over the floor. The contour was measured in 3 axial planes and the deviation compared to the design contour was less than $5 \mu \mathrm{m}$. The model with its transducer ports is shown in fig. 1.

\section{B. Flow Conditions}

The maximum total pressure and the minimum total temperature were $5.6 * 10^{6} \mathrm{~N} / \mathrm{m}^{2}$ and $230 \mathrm{~K}$, respectively. Before the incoming dry nitrogen flow is accelerated in a convergent-divergent nozzle to supersonic velocity it passes a settling chamber (with a diameter of $0.122 \mathrm{~m}$ and composed of a honeycomb/screens combination), a cross-section constriction and a bending tube section, where a side load measurement device can be inserted. In the current test setup a rigid dummy was mounted. The setup is sketched in fig. 2.. Total pressure and total temperature were measured between settling chamber and constriction.

\section{Instrumentation and Data Acquisition}

Up to 32 XT-154-190M Kulite pressure sensors were used to measure the wall pressure. The outer diameter of the sensors is $3.9 \mathrm{~mm}$ with a pressure sensitive area of $0.5 \mathrm{~mm}^{2}$ inside. The effective frequency response was limited to $330 \mathrm{~Hz}$ due to the length of the tubes connecting the pressure sensors and the transducer ports. The sensors were calibrated statically before mounting. The signal output was amplified by AS2 amplifiers, a DLR proprietary development, with a low-pass cut-off frequency of $160 \mathrm{~Hz}$. The analog signals were archived using a 12-Bit A/D converter with a sampling rate of $1 \mathrm{kHz}$.

\section{Optical Flow Investigations}

The free-standing horizontal test position offers simultaneous optical access. The exhaust jet was investigated with a Color Schlieren setup based on the dissection technique developed by $\mathrm{Cord}^{2}$ and improved by Ciezki ${ }^{1}$. Twelve 60x60 images per test were taken with a Hasselbad EL 500 camera and subsequently digitalized. The flow separation and its re-attachement were investigated using IR techniques and back flow frosting (BFF), where hoar frost detects the separation line. ${ }^{11}$

\section{E. Test Cycles}

The typical test profile was a mixture of smooth ramps and stationary plateaus (fig. 20). Smooth ramps to achieve quasi stationary conditions for separation detection at each pressure position and stationary plateaus to achieve Schlieren images. 


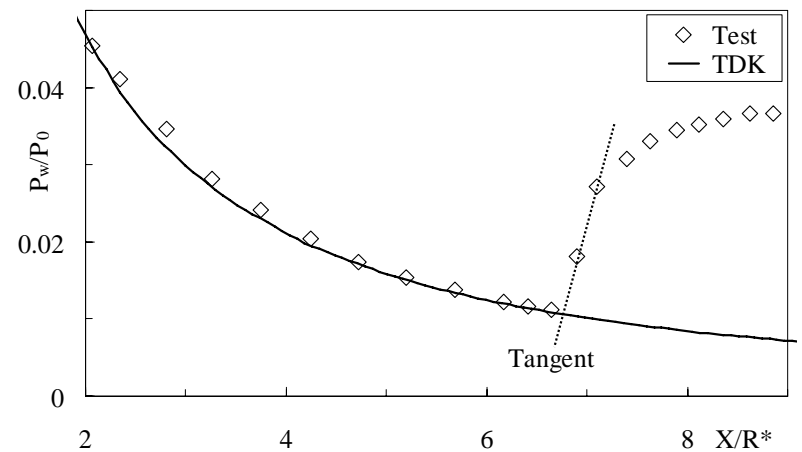

Figure 3. Non-dimensional wall pressure, $L / R^{*}=9$, $\mathrm{NPR}=25.25$

\section{F. Data Evaluation}

The comparison of non-dimensional wall pressure data and non-dimensional vacuum wall pressure profile obtained by a numerical MOC analysis gives the location of the flow separation (fig.3). The intersection of the vacuum wall pressure profile and a tangent along the steepest wall pressure gradient marks the separation location and therefore its corresponding wall Mach number.

A developed software allows to overlay 3D nozzle contour grids to any images given. The grids can be rotated in 3 axial directions, zoomed and deformed. Calibrated with a well known acrylic glass template the flow field can be measured (fig. 4).

\section{Discussion of Experimental Results}

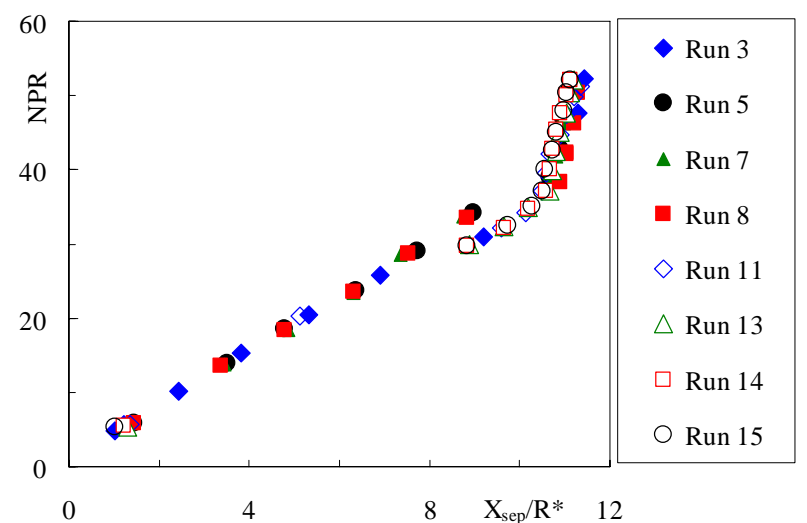

Figure 4. Separation location, $L / R^{*}=12$.

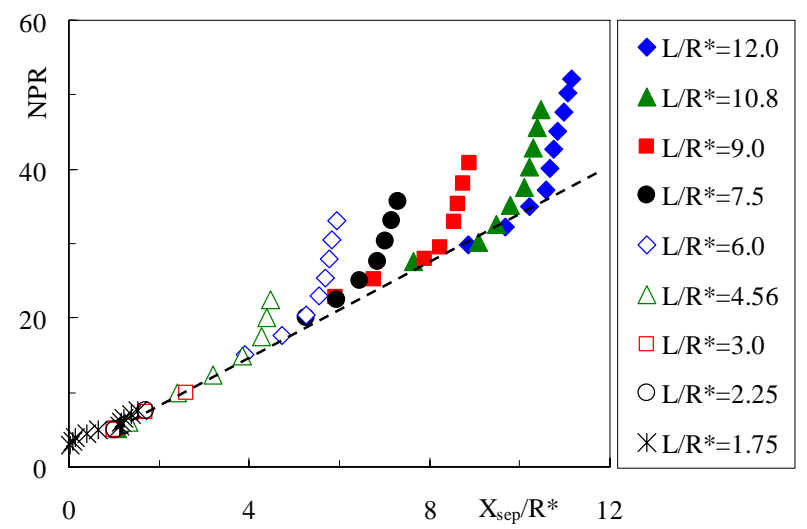

The test objective was to investigate the flow pattern in TIC nozzles at low NPR. For such conditions the oblique shock and the Mach disk are located inside the nozzle. So it was decided to cut the nozzle step by step from a length to throat radius ratio of $\mathrm{L} / \mathrm{R}^{*}=12$ down to $\mathrm{L} / \mathrm{R}^{*}=1.75$. The obtained flow patterns were compared to study the influence of the nozzle length on separation location and Mach disk position.

\section{A. Separation Location}

Separation location and NPR show a linear correlation (fig. 4). The discontinuity around NPR $=30$ is caused by nitrogen condensation resulting in a delayed separation. As the separation zone reaches the nozzle exit (see fig. 19a) a furthermore increased NPR only results in a compressed separation zone, indicated in fig. 4 by the steeper gradient near the nozzle exit.

Fig. 5 gives the separation locations of all tested nozzle lengths. The data follow a linear trend as long as the flow really separates. An influence of the nozzle length on separation location can be neglected

\section{B. Mach Disk Location}

A comparable linear correlation is given for the axial Mach disk position (fig. 13) and an influence on the axial Mach disk position can be neglected too.

Figure 5. Averaged separation locations. 


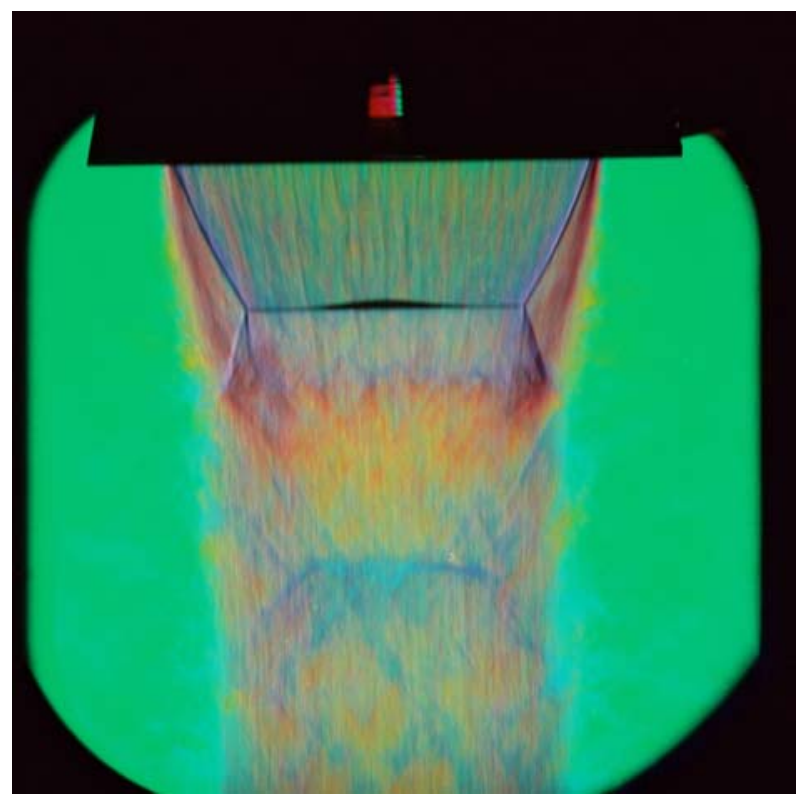

Figure 6a. $L / R^{*}=9$; NPR 30; $H_{\text {Mach }}=1.7 \mathrm{~mm}$

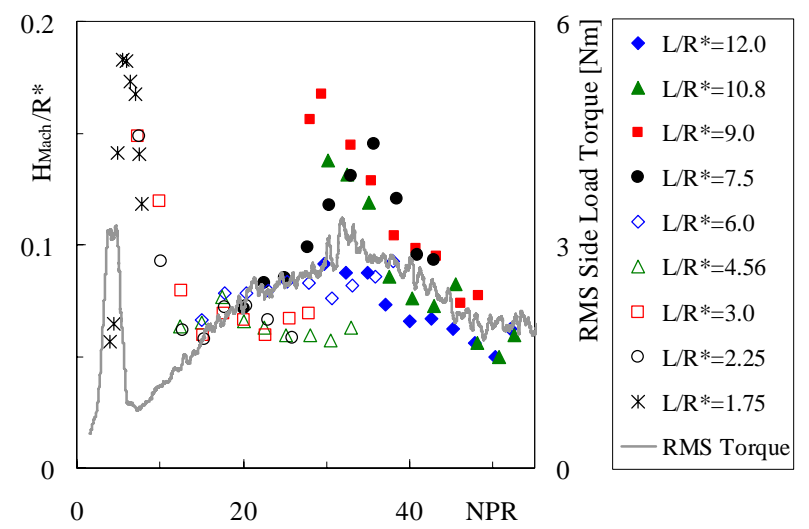

Figure 7. Averaged Mach disk curvature $\mathbf{H}_{\text {Mach }}$.

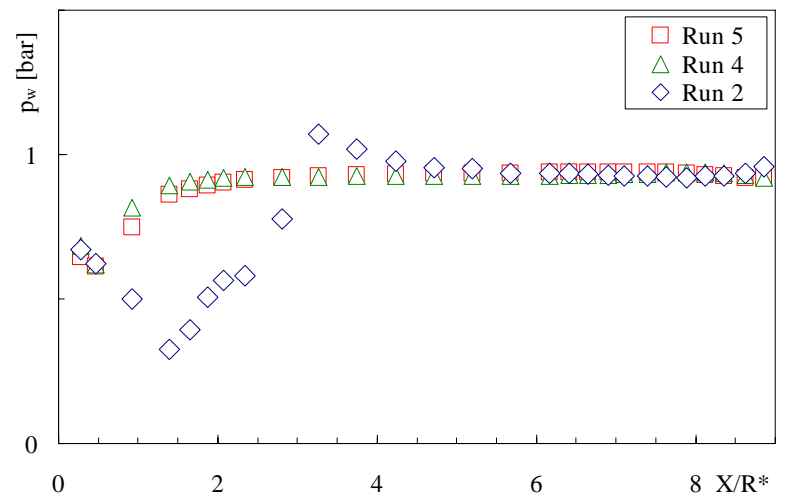

Figure 8. Wall pressure, $L / R^{*}=9, N P R=4.88$.

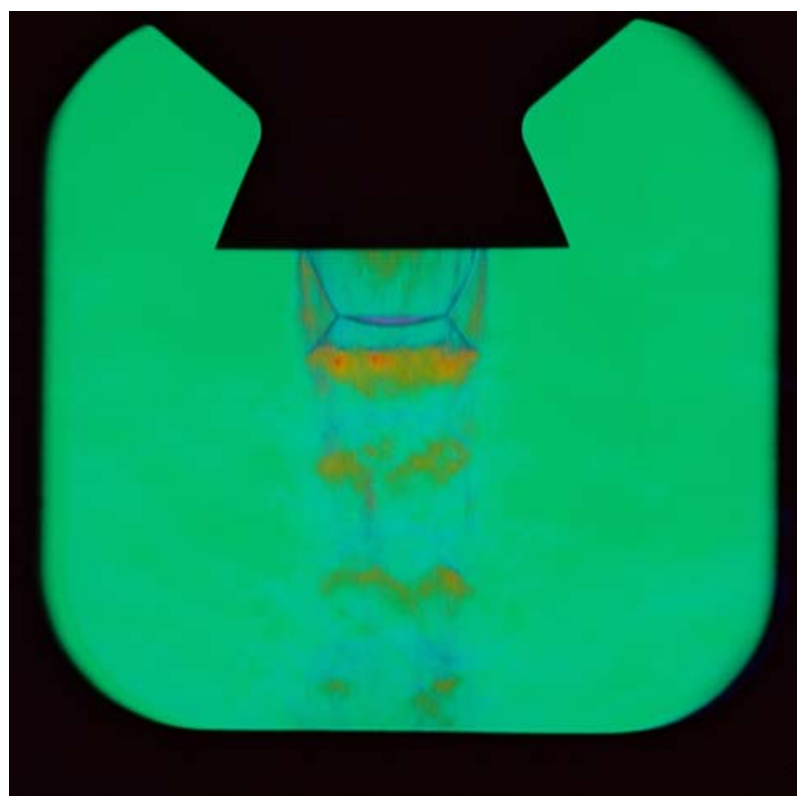

Figure 6b. $\quad L / R^{*}=1.75 ; \mathrm{NPR} \sim 5.5 ; \mathrm{H}_{\text {Mach }}=1.8 \mathrm{~mm}$

\section{Mach Disk Curvature and Side Loads}

As discussed the obtained results are representative. CFD investigations predicted clearly bowed concave mach disks but in the Schlieren images only Mach disks with a slight curvature limited to the centre were found (fig. 6a). Fig. 7 gives the height of the curvature related to the unaffected base as a function of NPR. A maximum is reached for a NPR of 30 to 35 . This distribution correlates with the side loads measured for nozzles of full length as shown in the graph.

A second peak is given for NPR below 10. But here the concave shape turns to a convex one (fig 6b). Therefore a concave bowed Mach disk, as obtained by former CFD investigations, can be excluded as the origin of a redirected flow towards the wall resulting in a side load peak.

\section{Partial Re-attached Flow and Tilted Mach Disk}

Nevertheless partial re-attached flows could be documented with wall pressure measurements for NPR $\sim 5$ (fig. 8). As the occurrence of this pattern is in circumferential direction randomly distributed only some of the performed test runs caught the re-attached flow condition. The wall pressure profile of run 2 with its downstream shifted separation and wall pressures above ambient conditions is typical for re-attached flows as it is e.g. known for restricted shock separation (fig. 19b).

For restricted shock separation a recirculation with lower wall pressures compared to the ambience forms out (fig. 19b). As a consequence the separation front jumps downstream. Entirely different for increasing low NPR: First the separation jumps and afterwards the 


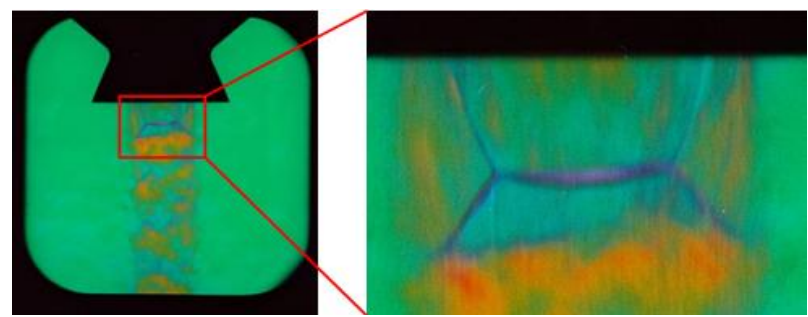

Figure 9. Tilted Mach disk; $L / R^{*}=1.75 ; \mathrm{NPR}=4.93$

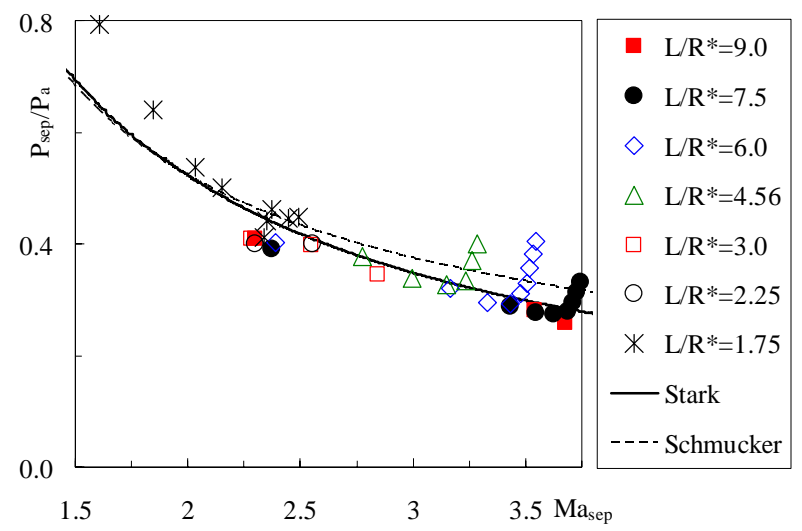

Figure 10. Separation and wall Mach number

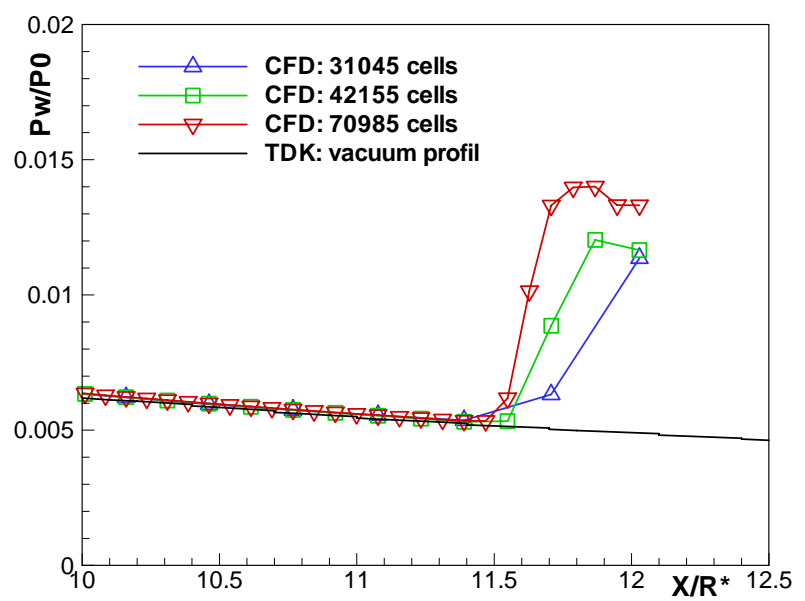

Figure 11. Comparison of wall pressure, NPR $=60$ flow re-attaches. This could be shown with a very short nozzle where suction effects of re-attached flows can be excluded (fig. 9). Consequently the Mach disk is tilted and redirects the flow towards the (imaginary) nozzle wall. This process is locally limited (fig. 22) and reversible as the NPR is further increased (fig. 21). It's a close-limited instability in separation behavior.

Figure 10 is a more conventional plot where the separation pressure is given as a function of the wall Mach number. The $\pi$-criteria $\mathrm{p}_{\text {sep }} / \mathrm{p}_{\mathrm{a}}=\pi /\left(3 \mathrm{Ma}_{\text {sep }}\right){ }^{12}$ reproduces the separation pressure data for wall Mach numbers $>2.5$ quite well. This trend gets lost for wall Mach numbers $<2.25$. The intermediate range around a wall Mach number of 2.4 (representing NPR =5) is unstable.

\section{Comparison with Complementary CFD Investigations}

\section{A. Numerical Method}

Numerical simulations have been performed using the $\mathrm{TAU}^{5}$ code developed by DLR. The Reynolds averaged Navier-Stokes equations are solved using a finite volume technique. The code can handle unstructured, structured, and hybrid meshes made of tetrahedral, pyramids, prisms, and hexahedra. The AUSMDV solver was chosen for the current study from the different available central and upwind schemes. It is accurate to the second order of space. The temporal gradients are discretized by a three stage Runga-Kutta scheme. Acceleration techniques like local timestepping, multigrid, and residual smoothing are available and have been used for the current simulations. Several one- and two-equations turbulence models are implemented into the TAU code. The computations have been carried out with the Spalart and Allmaras ${ }^{10}$ model.

The DLR-TIC with a length of $\mathrm{L} / \mathrm{R}^{*}=12.0$ was computed on an axi-symmetric hybrid mesh. The nozzle walls are considered to be adiabatic. The wall distance of the first cell of the structured boundary layer mesh is kept fine in order to have a $\mathrm{y}^{+}$value less then 1 . For the outer boundaries the farfield interpolation with ambient conditions was used. The values of the pressure inflow were varied to have NPR from 60-5.

\section{B. Validation}

The influence of grid refinement was studied for NPR $=60$ on three different meshes (fig. 11). The number of nodes along the nozzle wall was halved for each coarser grid. The computed results are compared with the wall pressure distribution obtained with TDK for vacuum conditions. The wall pressures of the CFD and TDK are in a good agreement until the separation. TDK is not able to predict flow separation. Experimental data are not available for the pressure ratio of 60 . The position and the gradient of the pressure rise are slightly depending on the grid size.

The following computations were carried out on the fine grid. This ensures the best separation prediction. Starting from NPR = 60 every pressure ratio was computed using the converged solution of the previous computation. 


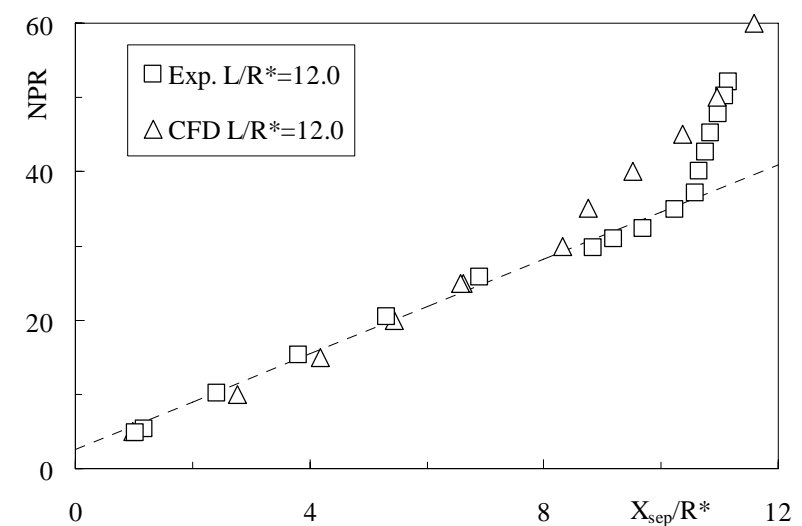

Figure 12. Separation point location

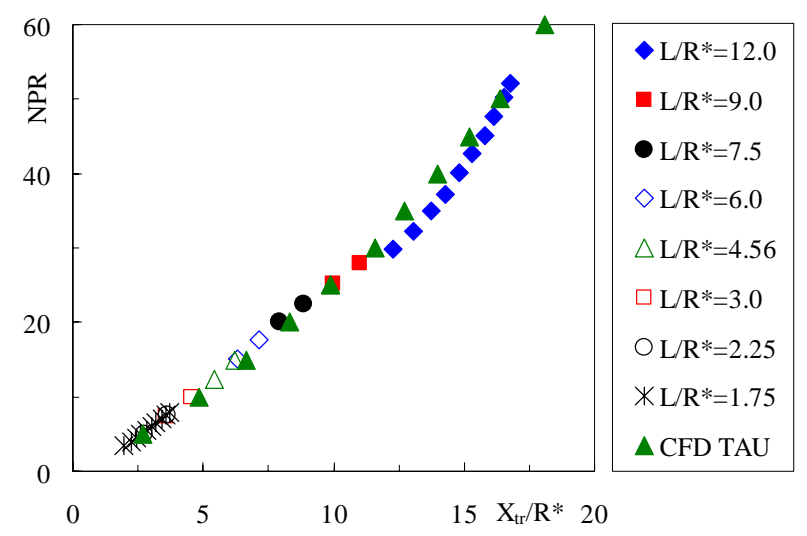

Figure 13. Axial position of the triple point

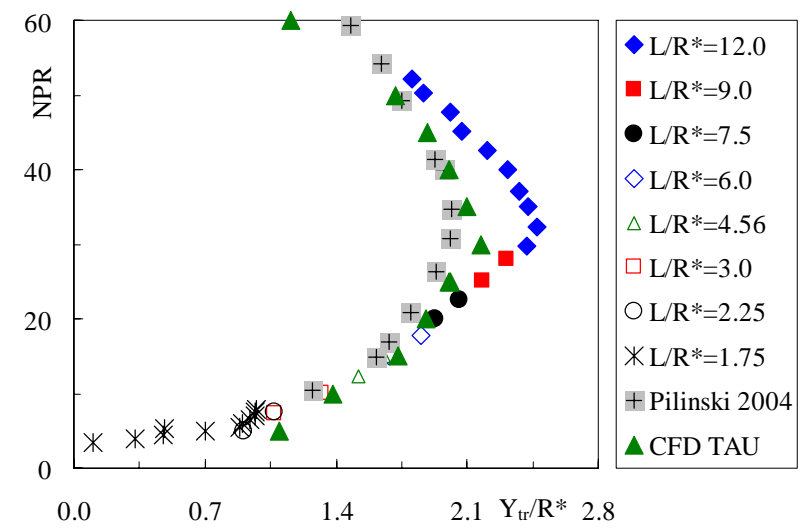

Figure 14. Radial position of the triple point

\section{Flow separation}

The separation locations of computed and experimental data are compared in figure 12. For pressure ratios of NPR $<30$ the computed separation positions are in a good agreement with the experiments. For pressure ratios greater then 30 , the computed separation positions differ from the experiments and the linear correlation. In this region condensation affects the wall pressure as already mentioned and therefore the separation location. The numerical simulations using the perfect gas assumption does not capture the condensation. But the effect of condensation is negligible compared to the maximum offset of $15 \%$ at $\mathrm{NPR}=35$. The influence of the ambience which affects the separation behavior close to the nozzle exit in the experiments can be found in the computations, too. The backflow behind the separation zone might be overpredicted which results in a separation further upstream. At NPR $=50$ the separation point is near the exit. The computations are again closer to the experiments.

\section{Mach disk}

For each pressure ratio the position of the computed triple point was estimated from the visualized solution. Its position is compared with positions measured from the Schlieren images. Below NPR $=30$ the shock system is inside the nozzle. Thus, for a nozzle length of $\mathrm{L} / \mathrm{R}^{*}=12.0$ the available data are limited. The comparison is continued with results from the piecewise truncated nozzle. The values were chosen to have a consistent prosecution for lower pressure ratios with an increment of $\triangle \mathrm{NPR}=2.5$.

The axial distance of the triple point correlates with the separation position. The discrepancies in the separation position prediction in the range of NPR $=30$ 50 lead to a disappointing agreement in the triple point positions (fig. 13). For pressure ratios below 30 the computed axial positions are in the range of the experimental results, although the values are from nozzles with shorter length. That underlines the previous observation that besides the ambient effect the truncation has no significant influence on the flow development.

The numerical estimated radial position of the triple point which is equivalent to the radius of the Mach disk already differs for NPR $>25$ from the experiments (fig. 14). The predicted Mach disk radius is up to $10 \%$ smaller then in the experiments. The results are also compared with computed radii obtained by Ref. 9 on a scaled version of the DLR-TIC. The deviation between these CFD results is small. Both are following the trend of a maxima Mach disk size at NPR $=30$ but are less shaped.

\section{E. Flow field}

We have proofed that the computations on the nozzle length of $L / R^{*}=12.0$ are also valid for shorter nozzles. The experimental test setup gives us the opportunity to compare the computed flow field with Schlieren images from shorter nozzles at very low pressure ratios. Figure 15 shows a satisfying agreement of the overexpansion shock. The 


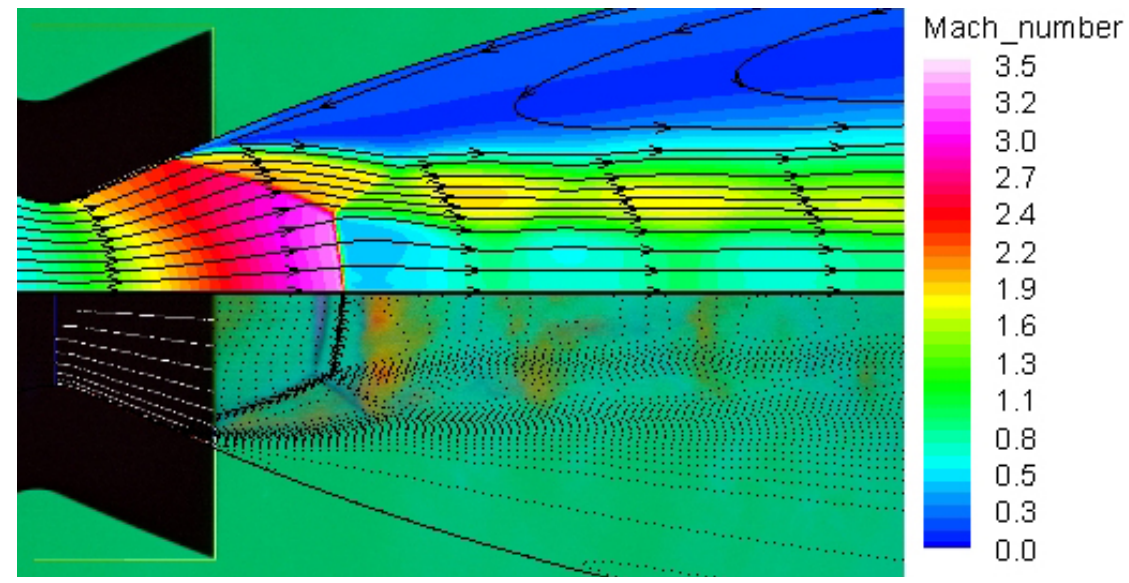

Figure 15. Schlieren image, $L / R^{*}=1.75$ and computed Mach distribution $L / R^{*}=12.0$ for $N P R=5.5$

axial position of the Mach disk is slightly overpredicted, but the convex shape is well reflected. Indications for a reattached flow could not be found in the steady state simulations. A small vortex downstream of the Mach disk is only present in the computations of NPR $=25-35$. The appearance of vortices could not be verified by the experiments.

The vortex influences the curved shape of the Mach disk. Thus, the highest deflections of the Mach disk were computed in the range of NPR $=20-40$ and they are slightly higher then in the experiments.

\section{Conclusion}

Test where carried out to examine the flow field in a truncated ideal contour nozzle for low NPR. The flow field was investigated by cutting a nozzle step by step. Wall pressure data and Schlieren images were obtained. It was shown that the data are unaffected by the length of the separated backflow. The data are reliable.

For NPR $<10$ a convex shaped mach disk was found and not a concave one as predicted by CFD investigations. ${ }^{6,8,9}$ A concave shaped Mach disk being responsible for re-attached flows could be excluded. For NPR $>20$ a slight bowed concave Mach disk was found. The curvature is limited to the centre. Its height trend correlates with measured side loads. An unstable situation is given for NPR around 5 and causes a temporary jump of the separation location. As a consequence the Mach disk is tilted and redirects the flow towards the wall. The separation characteristic for NPR $>5$ can be reproduced using Stark's $\pi$-criteria. ${ }^{12}$

The computations compare well with the separation and the triple point positions for NPR $<30$. The shape of the Mach disk is also well predicted even for low pressure ratios. Indications for a reattached flow and a strongly bended Mach disk at NPR 5 could not be found.

\section{Acknowledgments}

The authors would like to thank their colleagues of the 'Flow Separation Control Device' group ${ }^{4}$ (CNES, DLR, EADS ST, ESTEC, ONERA, LEA at Portiers, Snecma, Volvo Aero Corporation) for fruitful scientific exchanges.

Special thanks go to Christian Böhm (head of P6.2), Helmut Ciezki and David Zerjeski for outstanding teamwork.

\section{References}

${ }^{1}$ Ciezki, H., "Entwicklung eines Farbschlierenverfahrens unter besonderer Berücksichtigung des Einsatzes an einem Stosswellenrohr,” Diploma Thesis, Technical University Aachen, Germany, 1985.

${ }^{2}$ Cords, P., “A High Resolution, High Sensitivity Colour Schlieren Method,” S.P.J.E. Journal, Vol. 6, 1968.

${ }^{3}$ Frey, M., "Behandlung von Strömungsproblemen in Raketendüsen bei Überexpansion“, Ph. D. Thesis, Universität Stuttgart, 2001.

${ }^{4}$ Frey M. et.al., “Joint european effort towards advanced rocket thrust chamber technology,” 6th International Conference on Launcher Technologies, November 2005

${ }^{5}$ Gerhold, T., Galle, M., Friedrich, O., Evans, J., "Calculation of complex three-dimensional configurations employing the DLR-Tau-code,” $35^{\text {th }}$ AIAA Aerospace Sciences Meeting \& Exhibit, AIAA 97-0167, Jan. 1997

${ }^{6}$ Kwan W., and Stark R., “Flow Separation Phenomena in Subscale Rocket Nozzles”, AIAA Paper 2002-4229, 2002.

${ }^{7}$ Kronmüller H., Schäfer K., Zimmermann H., Stark R., “Cold Gas Subscale Test Facility P6.2 at DLR Lampoldshausen”, 6th Symposium on Propulsion for Space Transportation of the XXIth century, Versailles, 2002.

${ }^{8}$ Nasuti F., Onofri M. and Pietropoli E., "Prediction of shock generated vortices in rocket nozzles,” AIAA Paper 2005-317, 2005. 
${ }^{9}$ Pilinski C. and Nebbache A., "Flow separation in a truncated ideal contour nozzle,” Journal of Turbulence, Vol.20, No.3, 2004

${ }^{10}$ Spalart, P. R., and Allmaras, S. R., “A One-Equation Turbulence Model for Aerodynamic Flows,” 30 th AIAA Aerospace Sciences Meeting \& Exhibit, AIAA 92-0439, Jan. 1992

${ }^{11}$ Stark R., Kronmüller H., Zerjeski D. and Wagner B., “Advanced Flow Visualisation Techniques in Cold Gas Subscale Nozzles, a Comparison”, AIAA Paper 2003-5180, 2003.

${ }^{12}$ Stark R., “Flow Separation in Rocket Nozzles, a simple Criteria”, AIAA Paper 2005-3940, 2005.

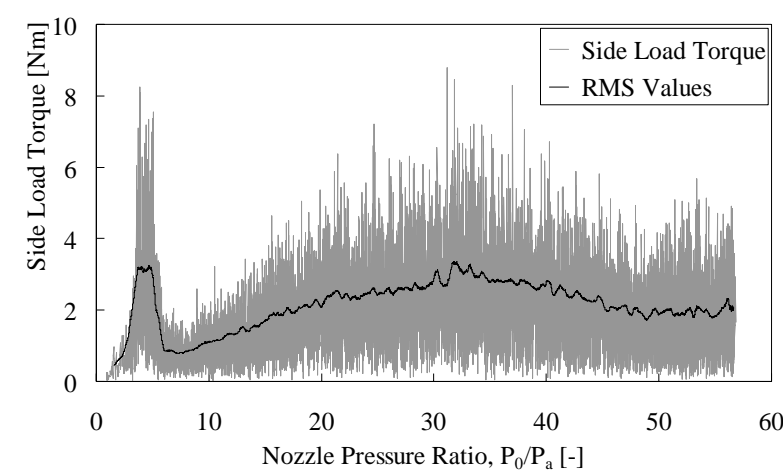

Figure 16. Typical side-load behavior for DLR TIC nozzle.

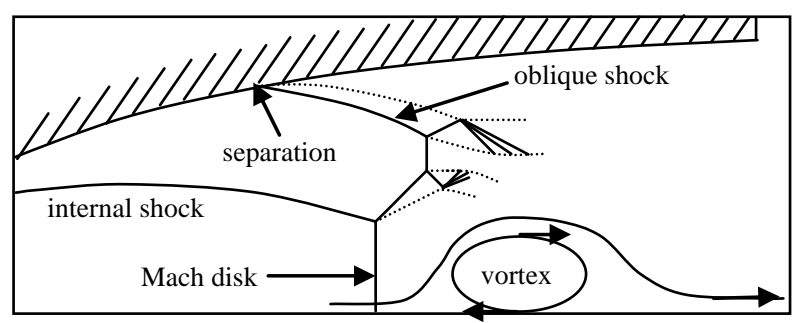

Figure 18a. Cap Shock pattern with FSS for the TOP nozzle.

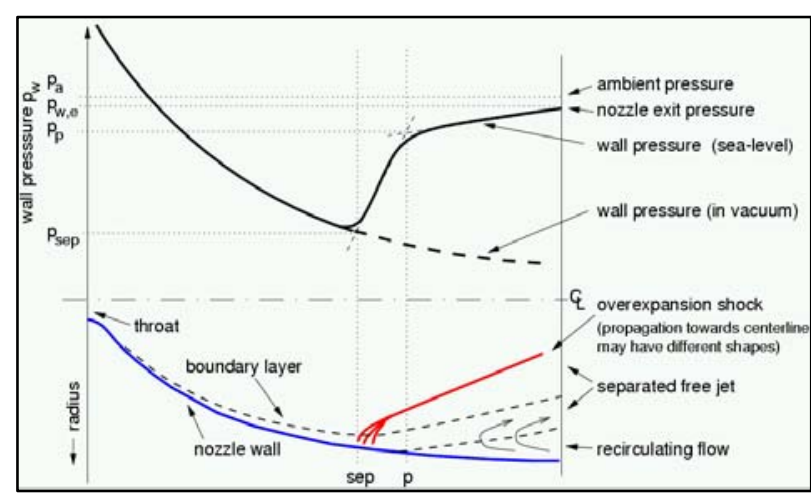

Figure 19a. Free shock separation, taken from [3]

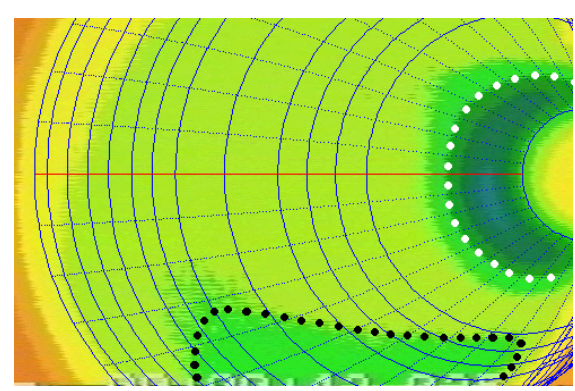

Figure 17. IR image showing re-attached flow during startup, NPR=4.7. Separation: white dots. Re-attached flow: black dots.

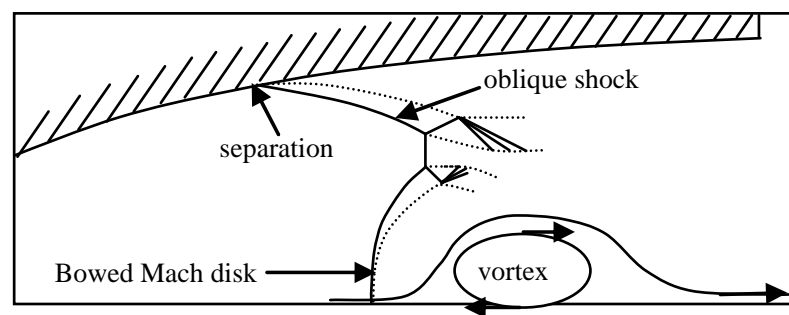

Figure 18b. Curved Mach disk with FSS for the TIC nozzle.

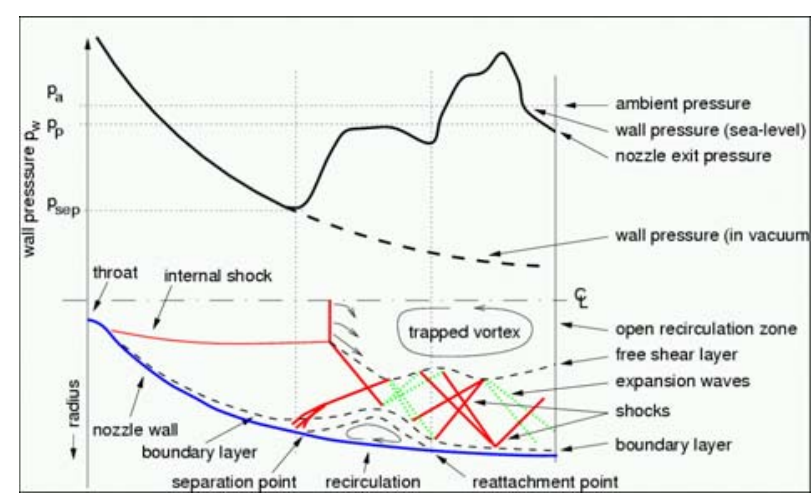

Figure 19b. Restricted shock separation [3] 


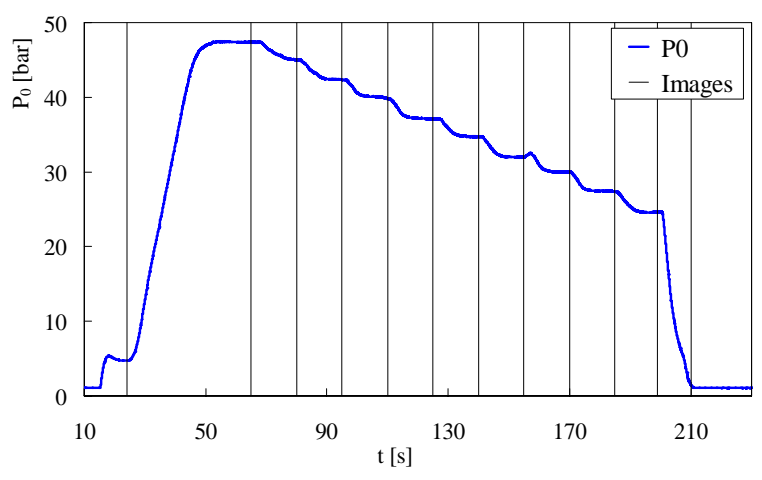

Figure 20. Typical stagnation pressure profile.

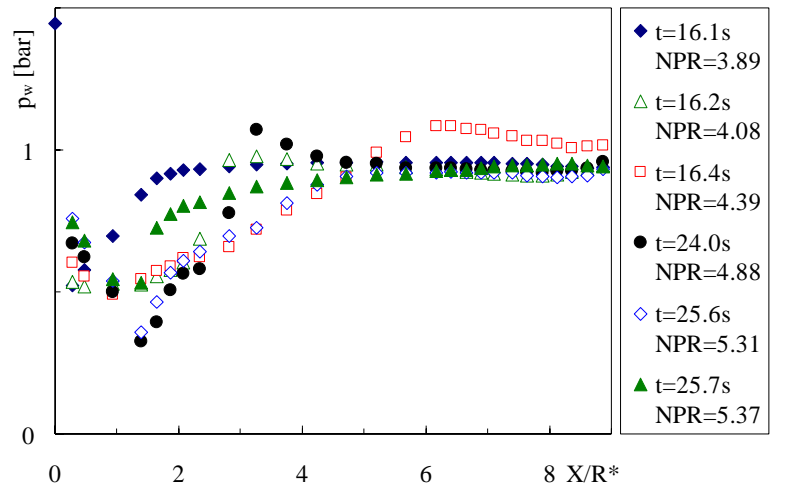

Figure 21. Wall pressures, $L / R^{*}=9$, increasing NPR

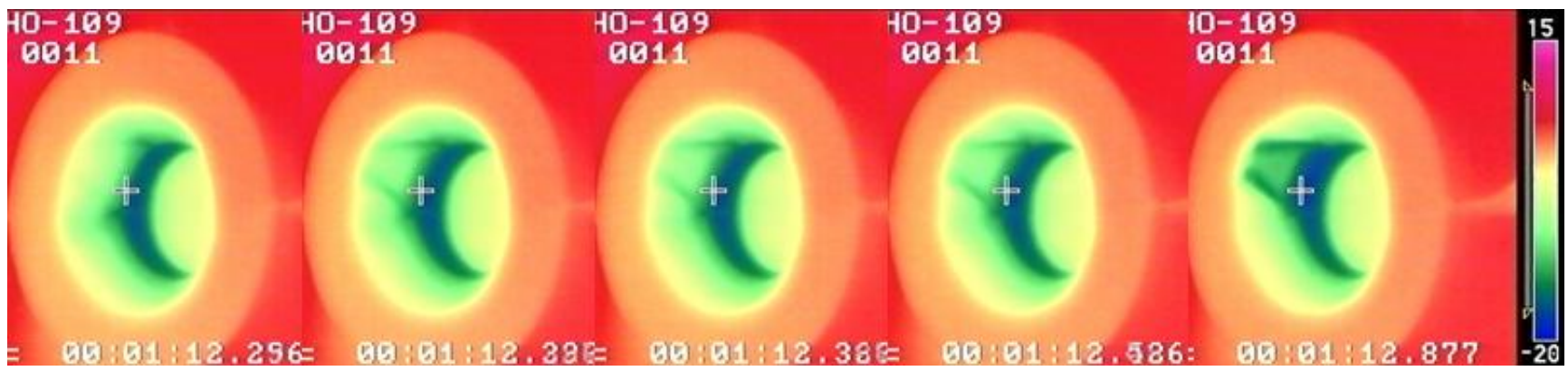

Figure 22. IR images, $L / R^{*}=1.75$ and $N P R=4.6$ 